\title{
Cambios periféricos en el desarrollo de la teoría de la selección natural*
}

Non-Substantial Changes in the Development of the Theory of Natural Selection

Daniel Blanco ${ }^{\dagger}$

\begin{abstract}
Resumen
Esta contribución se aproxima al desarrollo transtemporal de las teorías científicas mediante un estudio de caso particular: el modo en que la modificación diacrónica del conjunto de aplicaciones pretendidas de la teoría de la selección natural afecta (o no) a su identidad. La perspectiva metateórica elegida involucra rudimentos del enfoque estructuralista de las teorías, y la discusión incluye tanto la presentación jerárquica del constructo teórico como la tematización de un debate entre los autores de la teoría en cuestión, Alfred Wallace y Charles Darwin. Se ilustra así la genidentidad de esta teoría particular ante cambios pragmáticos relativos a sus aplicaciones concretas.
\end{abstract}

Palabras clave: aplicaciones pretendidas - ejemplar - teoría de la selección natural

\begin{abstract}
This contribution is about the development of scientific theories in time through a particular case study: the way in which the diachronic modification of the set of intended application of the theory of natural selection impacts (or not) in its identity. The chosen meta-theoretical approach involves some rudiments of the structuralist view of theories, and the discussion includes both the hierarchical presentation of the theory as a debate between Alfred Wallace and Charles Darwin, the co-authors of the theory. Thus, we illustrate the genidentity of this particular theory when it faces pragmatic changes in its concrete applications.
\end{abstract}

Keywords: intended applications - exemplar - natural selection theory

\footnotetext{
* Recibido: 23 de diciembre de 2015. Aceptado con revisiones: 15 de marzo de 2016.

† Universidad Nacional del Litoral/Universidad Autónoma de Entre Ríos, Argentina. Para contactar al autor, por favor, escribir a: danielblanco.fb@gmail.com.

Metatheoria 6(2)(2016): 81-93. ISSN 1853-2322. eISSN 1853-2330.

(c) Editorial de la Universidad Nacional de Tres de Febrero. Publicado en la República Argentina.
} 


\section{Introducción}

La relación entre historia de la ciencia y filosofía de la ciencia se alimenta bidireccionalmente, y numerosas veces el estudio serio de una aparece iluminando las pertinencias de la otra, conjuntos estos cuyas fronteras no son siempre claras. Esta contribución, de tinte historiográfico, realizado a la luz de intuiciones propias de la "concepción estructuralista" de las teorías científicas (Balzer, Moulines \& Sneed 1987), cuyos rudimentos supondremos, pretende mostrar aspectos de la evolución o desarrollo diacrónico de la teoría de la selección natural (infra, TSN), en particular con su aspecto genidéntico. Mostraremos cómo la incidencia de algunos cambios reconocibles en su "periferia", no afectan la conservación transtemporal de su identidad por no comprometer la estabilidad de componentes considerados esenciales. Por supuesto, hay más de un tipo de estas variaciones periféricas que el historiador de la ciencia puede contribuir a especificar (Stegmüller 1976, p. 226), muchas de las cuales dan lugar a distintos tipos de cambio (Moulines 2011), y este no pretende ser un estudio exhaustivo de todas éstas. En cambio, nos vamos a concentrar en identificar algunas modificaciones en la extensión del conjunto de aplicaciones pretendidas o intencionales (intended applications), I, o, más bien, de un parte del mismo (el subconjunto $I-I_{0}$, siendo $I_{0}$ el conjunto de aplicaciones exitosas paradigmáticas o ejemplares de la teoría, en el sentido kuhniano).

La presentación tiene la siguiente forma:

(1) Primeramente damos un muy breve esbozo de la estructura de TSN, subrayando algunos aspectos pertinentes señalados por algunas de las reconstrucciones disponibles.

(2) Posteriormente, y a los fines ilustrativos, tematizamos algunos pocos aspectos relativos al dominio de aplicación primitivo propuesto por Alfred Wallace y Charles Darwin. Como consecuencia colateral de esto, veremos un caso que ilustra los buenos servicios que un análisis sistemático puede aportar a los intereses históricos; en esta oportunidad, a la interpretación de una conocida discusión entre los aludidos co-fundadores.

(3) Por último, tematizamos un estudio relativamente reciente (Takahashi et al. 2008) que parece atentar contra un caso ejemplar de TSN (en particular, de uno de sus elementos teóricos especializados, en este caso el correspondiente a la teoría de la selección sexual), evaluando algunas de sus consecuencias.

\section{Estructura jerárquica de TSN}

Darwin, al igual que otros naturalistas, tenía en claro que la responsabilidad de la emergencia de la novedad estructural y de su preservación en la descendencia de los organismos que la portan (y que logran reproducirse) caía sobre cierta teoría de la herencia que menciona desde muy temprano en su carrera (ver Manuscrito E48; Cornell 1987, p. 404), pero de la cual no dijo demasiado sino hasta fines de la década de 1860 (Darwin 1868, vol. II, pp. 357-404). La selección natural, por su parte, sería la responsable de la preservación y proliferación de estas novedades gracias a la reproducción diferencial de sus portadores.

Aunque en muchos abordajes al tema se llama TSN a las tres cosas en conjunto (emergencia de la variación, selección, y transmisión hereditaria), parece sensato tener en cuenta esta distinción a la hora del análisis. Siempre conviene saber si cuando se habla de "selección natural" nos referimos exclusivamente al proceso selectivo, sin atender a la teoría hereditaria involucrada (como por momentos hacía Darwin), o directamente a la explicación de fenómenos evolutivos que viene proporcionada por TSN (lo cual no excluye el que otras teorías pudieran dar cuenta de ciertos casos de evolución). 


\subsection{Vacuidad empírica y jerarquía teórica}

En los análisis metateóricos disponibles relativos a la teoría de la selección natural, dos cuestiones han sido puestas sobre la mesa con relativa frecuencia:

(1) En primer término, en una crítica que data (al menos) desde los escritos de Mivart (1898, p. 272), se sospecha del carácter cuasi-tautológico, o analítico de la misma. Como consecuencia de ello, en algunos círculos (definitivamente no en todos) incluso se llegó a dudar de su cientificidad, en una revuelta que tuvo su impacto en debates político-institucionales que a su vez derivaron en varias ocasiones en litigios legales.

Estas sospechas vienen dadas básicamente por dos razones distinguibles: (a) por un lado, por la adopción de parte de Darwin (a partir de la $5^{a}$ edición de On the Origin of Species) de la conocida expresión spenceriana "supervivencia del más apto"; y, por otro lado (y a veces ligado con lo anterior), (b) por cierta supuesta laxitud de la teoría a la hora de incorporar elementos disímiles a su dominio de aplicación. Por ejemplo, la selección natural puede dar cuenta de la proliferación no sólo de la novedad sino también de la estabilidad (Darwin 1872, p. 154). Además, las novedades favorecidas pueden darse en cualquier dirección: desde la especialización y complejización de los rasgos, hasta su atrofia e incluso su desaparición (Darwin 1872, p. 155). Esta notable fertilidad, es tomada por muchos como un exceso atribuible a la analiticidad, más que como una virtud a destacar.

Aunque el carácter analítico de la teoría (o de parte de ella) puede discutirse (ver Lorenzano 2007, Falguera 2012), creo que es relativamente sencillo ver qué es lo que produce estas dificultades: cada vez que nos preguntamos cómo es que la selección natural explica situaciones tan dispares, la respuesta recurrente es que hay "algo", hay una ventaja relativa por parte de los individuos con cierta configuración en sus rasgos a circunstancias contingentes que vuelve esperable al estado del que se pretende dar cuenta (ver Darwin 1872, p. 148).

Esta es una respuesta vaga porque la primera reacción ante el pedido de una explicación para el favorecimiento de un individuo con ciertos rasgos en una región -favorecimiento en términos reproductivos- es que simplemente existe una ventaja particular en comparación con otros. Pero no se especifica en qué consiste esa ventaja. Claramente, esta "cierta" ventaja o beneficio vincula a la adecuación, y/o al desempeño y/o a la utilidad de algún rasgo particular con el éxito diferencial en la reproducción del individuo que lo porta. Pero, insistimos, la ventaja no es explicitada. ${ }^{1}$

Notemos cómo aparece esto en boca de un autoproclamado ultradarwinista:

La evolución es notablemente eficiente cuando se trata de sacar de escena los accidentes inútiles, de modo que si encontrásemos un patrón persistente de actividades [...] costosas, podemos estar bastante seguros de que con ello alguna cosa está obteniendo un [cierto] beneficio, que ha de notarse al hacer el único balance que le interesa a la evolución: el de la reproducción diferencial. (Dennett 2007, pp. 88-89, énfasis nuestro)

Si algún rasgo "prospera", multiplicando su presencia a lo largo de las generaciones, inmediatamente se piensa en una ventaja particular que ese rasgo otorga a aquellos individuos que lo poseen, y se confía en ello aun antes de que la misma sea identificada. El punto es que no hay ningún experimento respaldando esa confianza inicial. De hecho, si esto es efectivamente lo único involucrado en TSN, entonces no parece ilegítimo que se generen sospechas. Con todo, veremos que en realidad TSN sí contiene en su estructura elementos teóricos que permiten efectuar la especificación reclamada.

(2) En segundo término (y esto no está necesariamente divorciado de lo anterior), se sostiene que lo que en general se denomina TSN es en realidad un aglomerado de teorías que puede representarse más adecuadamente como una colección jerárquica de elementos relacionados entre sí. Cuál es la

\footnotetext{
${ }^{1}$ El éxito reproductivo no se mide en términos de lo numerosa de la prole de una pareja determinada, sino más bien en términos de lo "promisorio" de la misma. Un escaso número de descendientes puede ser un mejor "ajuste" a las circunstancias que uno mayor. En resumen: no se puede saber a priori qué número en la prole es el más adecuado. Incluso aquí, otra vez, aparece la capacidad de la teoría para incluir aplicaciones exitosas: sea cual fuere el número concreto que observamos, existe para éste una explicación en el marco de la teoría.
} 
naturaleza de esa relación, depende de la perspectiva metateórica de los autores que reconocen esta estructura.

Estas dos cuestiones (la aparente vacuidad empírica y el carácter jerárquico de la teoría ${ }^{2}$ ) aparecen íntimamente vinculadas en algunas reconstrucciones sincrónicas de la teoría, cuestión a la que dedicamos la siguiente sección.

\subsection{El carácter jerárquico en (algunas de) las reconstrucciones disponibles}

John Endler, en su Natural Selection in the Wild (Endler 1986, p. 9), reconoce el carácter estratificado de la teoría, distinguiendo dos tipos de selección natural, la sexual y la no sexual, siendo el aspecto relacionado a la "supervivencia" (como así también otros) un caso particular de la segunda. Además, Endler nota que a medida que se baja en esta estratificación, más utilidad presenta la teoría para atender o dar cuenta de casos específicos.

El autor sostiene que la razón para el fracaso en ver estas distinciones por parte de muchos autores se debe a que el mismo Darwin no lo explicó claramente en su obra cumbre, donde hay expresiones en las que:

(1) la selección natural aparece identificada en numerosas ocasiones con la "supervivencia de los más aptos" (Darwin 1872, pp. 80, 102, 134-135, 180, 471);

(2) la selección sexual -como consecuencia de (1)- parece tener una aplicación excluyente respecto de la selección natural (Darwin 1872, p. 44); y

(3) la selección artificial -también como consecuencia de (1)- aparece muchas veces al mismo nivel que la natural (Darwin 1872, pp. 39, 80-81, 137-138).

Endler (1986, pp. 3-15), en cambio, interpreta que la supervivencia es sólo una parte de la historia: la "lucha en la que sobreviven los más aptos" ya no es (a pesar de varias expresiones darwinianas) coextensiva con la selección natural.

La segunda reconstrucción que quiero recuperar corresponde a una contribución de Juha Tuomi de 1981 ("Structure and Dynamics of Darwinian Evolutionary Theory"), un trabajo que es continuación de otro que había escrito en colaboración con Erkki Haukioja en 1979.

Tuomi sostiene que una representación adecuada de la teoría es la de un (así llamado por él, y siguiendo parcialmente categorías bungeanas) "modelo dinámico de multi-nivel”, en el que aparecen subestructuras interconectadas que cuentan con diferentes niveles de abstracción. Tuomi identifica la porción más elevada como el núcleo duro (lakatosiano) del cual no es posible extraer predicciones, lo cual hace que resulte infalsable. En cambio, de las teorías más "específicas" derivadas de este centro fuerte, sí es posible generar consecuencias observacionales, y por lo tanto aquellas sí tienen contenido empírico y sí resultan falsables. ${ }^{4}$

Tuomi (1981, pp. 28-30) concluye con una interpretación kuhniana de su propuesta: en tiempos de ciencia normal, se da un dogmatismo pragmático que se manifiesta en la preservación de las partes más abstractas de la teoría, mientras que se admite el cuestionamiento a la adecuación de la aplicación de las porciones de los niveles inferiores a casos particulares.

En tercer lugar, he decidido repasar la reconstrucción ofrecida por Santiago Ginnobili (2006, 2010, Ginnobili \& Carman 2008), cuyo trabajo se efectúa desde la perspectiva estructuralista. El tratamiento jerárquico reaparece en este enfoque: tenemos porciones que rigen para todas las aplicaciones de la teoría (lo que desde el estructuralismo se llama ley fundamental, o ley del elemento teórico básico), y otras a las que sólo se apela para ciertas aplicaciones particulares (las leyes de elementos teóricos especializados).

\footnotetext{
2 La lista de autores que señalan lo primero es extensa (Scriven 1959, Popper 1963, Manser 1965, Beckner 1968, Caplan 1977, Castrodeza 1977, Peters 1976, Krimbas 1984, Reid 1985, Løvtrup 1987, 1990, Loehle 1988). El número de autores que apuntara lo segundo es menor, pero igualmente significativo, y sobre algunos de los cuales pasamos breve revista a continuación.

3 Las referencias a la obra de Darwin agregadas en respaldo a los comentarios de Endler, son mías.

4 Tanto en el artículo de 1979 como en el de 1981, Tuomi sostiene que la relación entre el núcleo duro y las subteorías relacionadas con él (la derivación aludida) es de inferencia deductiva (Tuomi 1981, pp. 23, 27).
} 
Así, en la reconstrucción de Ginnobili aparece una vez más en el estrato más elevado la porción de la teoría donde se encuentra la ley que relaciona inespecíficamente a la utilidad de los rasgos con la mejora en la reproducción diferencial de los individuos que lo portan. Además, propone que la vinculación entre ambas cosas (un rasgo que cumple con una función de cierto modo con la reproducción diferencial) es un aporte conceptual de TSN (candidato a ser incluido en el conjunto de términos TSN-teóricos), a la que llama "aptitud". 5

En esta presentación aparece nuevamente la teoría de la selección sexual en relación con la teoría de la selección natural, como hacía Endler. La teoría de la selección natural está enriquecida por sus sucesivas especializaciones, donde aparecen incluso dos tipos de selección (reconocidos por Darwin, ver Darwin 1872, pp. 44-55) como lo son la selección artificial metódica y la no metódica, o consciente o no consciente. Al descender de estrato, el ámbito de aplicación se va restringiendo debido a que la teoría va ganando en contenido empírico por aumentar la especificidad de la ley de cada elemento teórico a través de la incorporación de restricciones.

Esta red arbustiva es la que me resulta la perspectiva más convincente de las tres, en tanto que:

(1) reconoce el carácter abstracto de una porción de la teoría (concretamente, en la ley del elemento teórico básico de ésta) -rescatando la intuición de Tuomi;

(2) muestra cómo un grado de especificación o concretización creciente contribuye a volver empíricamente relevante a la teoría -rescatando las intuiciones de Endler y Tuomi-; y

(3) no requiere, fiel al sentir estructuralista, de una relación de implicancia deductiva entre un elemento de un estrato cualquiera y su especialización inferior.

Esto es satisfactorio a las problemáticas filosóficas por varias razones. El reconocimiento de una jerarquía en la teoría, es un paso necesario a la hora de posibilitar la distinción de sus elementos constitutivos de los que son sencillamente periféricos. Por esta razón, la distinción permite discriminar entre los cambios meramente accidentales (desarrollo o cambio dentro de una teoría ${ }^{6}$ ) y los esenciales (evolución o cambio de teoría), cosa que tematizaremos a continuación.

\section{Inestabilidad transtemporal del conjunto de aplicaciones}

\subsection{El conjunto $I_{0}$}

Como sabemos, el conjunto de aplicaciones (I) de una teoría particular cualquiera identifica a las cosas del "mundo" de las que pretende hablar según lo quiere la comunidad de pares que hace uso de ella, por lo que su extensión está determinada volitiva y pragmáticamente (aunque el ejercicio de esa voluntad aparece al menos parcialmente constreñida por los ejemplares de la teoría) por las pretensiones utilitarias de tales usuarios (Moulines 1986, pp. 299-305), y, por lo tanto, constituye un conjunto abierto susceptible de modificación (Moulines 1986, pp. 302-303; Stegmüller 1976, p. 224). El subconjunto $I_{0}$ de "ejemplares" $\left(I_{0} \subseteq I\right.$ ) (en sentido kuhniano) no está sujeto a la pérdida de elementos, si es que no se ha de afectar a la "personalidad distintiva" de la teoría. ${ }^{7}$

\footnotetext{
${ }^{5}$ En lenguaje natural, la ley reza: "En determinada población existen diferencias de aptitud entre distintos tipos de organismos que vinculan la posesión de ciertos rasgos particulares de un mismo tipo y que cumplen su función o una de sus funciones con determinada efectividad con el éxito reproductivo de tales organismos".

${ }^{6}$ Habitualmente, desde el estructuralismo metateórico se denomina a este escenario como "evolución teórica”. Defiendo la inclusión de esta novedad terminológica (trocar el uso habitual de "evolución" por el de "desarrollo") en tanto que "desarrollo" connota cambio ontogenético, es decir, un tipo de modificación transtemporal en el que la identidad de la entidad cambiante no está en juego. En cambio, "evolución", si bien no implica necesariamente cambio de identidad, no lo prohíbe. Por eso, cuando hablamos de modificaciones teóricas en tiempos de ciencia normal, es más adecuado utilizar "desarrollo teórico" en vez de "evolución teórica" (por la ambigüedad de este último concepto, equiparable en este sentido al de "cambio"). "Evolución" no excluye a los cambios revolucionarios, pero tales cambios excepcionales son sólo uno de los varios tipos de cambios reconocibles en la historia de las teorías (Moulines 2011).

${ }^{7}$ Los "ejemplares" son aquellas aplicaciones intencionales (es decir, aquellos elementos de $I$ ) que son a su vez explicaciones exitosas (es decir, aplicaciones intencionales que satisfacen los axiomas, $I_{0} \subseteq r(\mathrm{M})$ ), y, además son utilizadas como herramientas didácticas en tiempos de "ciencia normal" (en sentido kuhniano) a modo de "ejemplo" de aplicación exitosa a la cual otras aplicaciones se parecen.
} 
Aquí quiero plantear una dificultad no menor relacionada con lo difícil que resulta la identificación de aplicaciones exitosas en la arista "histórica" de TSN. ${ }^{8}$ Si bien en la literatura uno puede encontrar numerosos casos tomados de On the Origin of Species a modo didáctico y de "ejemplo" estandarizados (al cual otros casos se parecen) de aplicación exitosa de TSN, está claro que uno no puede juzgar "exitosa" a una aplicación en la que está involucrada, por ejemplo, el origen de una determinada adaptación, del mismo modo o con exactamente el mismo criterio con el que puede juzgar el éxito de una aplicación de una teoría no histórica.

Por supuesto, hay una arista no histórica de TSN, y es con ésta con la que los biólogos trabajan continuamente en el campo. En tal caso, no parece especialmente difícil dar con aplicaciones exitosas si es que efectivamente las hay. Pero debe quedar claro que esto difiere de corroborar la aserción vinculada al origen efectivo de una adaptación mediante la enorme acumulación de eventos de reproducción diferencial por ciertas razones específicas (ver Cleland 2002).

Como sea, los criterios que determinan el éxito o fracaso de una teoría al ser aplicada en un caso particular también son determinados pragmáticamente por la comunidad científica. El punto es que en un sitio se acepta una extrapolación, y en el otro no es necesario apelar a ella. Claramente, el criterio por el que se reconoce a una aplicación exitosa en un caso y en el otro no es (y/o no debería ser) el mismo.

\subsection{La disputa Wallace-Darwin respecto de la exclusión de elementos en "I"}

Hasta hoy, cuando se discute el protagonismo que ha de atribuirse a la selección natural a la hora de dar cuenta del proceso evolutivo, tanto los que abogan por un panseleccionismo irrestricto como los que pretenden reducir tal campo de aplicación a un dominio más restringido, citan a Darwin con el fin de posicionar al gran naturalista inglés respaldando su propia posición.

Pero, y para seguir la expresión de Kuhn (1962, p. 85), al momento del anuncio de TSN ¿cuál es el rango concreto de fenómenos naturales a los cuales se la aplicó?

En principio uno diría que el explanandum primitivo de la teoría es el origen de las adaptaciones, "hechos observados" que en este caso sería un tipo de rasgo cuya existencia fuera detallada incluso por los teólogos naturales que las explicaban apelando a la intervención de un diseñador inteligente (Darwin 1859, pp. 60-61, Romanes, 1882, p. 16). ${ }^{9}$ Ahora bien, la selección natural, ¿es responsable (o co-responsable junto con los factores hereditarios) de la existencia de todas las adaptaciones?

Es interesante notar que los co-fundadores de la teoría disintieron respecto del alcance de ese rango, cosa que se evidencia por la propensión diferencial entre ellos a quitar elementos del conjunto de aplicaciones. Esta disputa es bastante conocida, y tuvo lugar epistolarmente, en publicaciones posteriores a 1859, y -de parte de Wallace- en varias contribuciones a Nature.

Generalmente se propone que Wallace sostenía que la selección natural habría de aplicarse indiscriminadamente, mientras que Darwin no. El ejemplo más comentado es el fracaso de la selección natural para explicar el origen de ciertos rasgos propios del hombre, como el lenguaje (ver Berwick \&

Reconstrucciones anteriores de otras teorías muestran que entre los ejemplares suelen estar las aplicaciones intencionales primitivas de la teoría, pero tal cosa no es condición ni suficiente ni necesaria para ser considerado un miembro de $I_{0}$.

8 Esta no es una dificultad exclusiva de TSN, sino que más bien parece tratarse de un problema que enfrentan muchas teorías que abordan tópicos situados en un pasado irrecuperable. Muchas veces, como en inferencias en cuestiones macroevolutivas o en tectónica de placas, se apela al poder unificador de las explicaciones involucradas. En el caso que nos ocupa, el problema se parece más a la analogía que se hace entre los exitosos experimentos de Miller y Urey y la explicación para con el origen de la vida: la relación entre la hipótesis testeada en el laboratorio con la hipótesis "blanco" (del pasado) es complicadísima y lleva consigo numerosas presuposiciones especulativas (ver Cleland 2002).

9 La determinación de estas entidades es independiente de la teoría, y por lo tanto, de lo que de la TSN depende, son datos no controvertidos, una porción de su base empírica, o elementos TSN-no-teóricos porque su determinación la precede y no la necesita (ver Blanco 2008, 2011, Caponi 2011). Es difícil encontrar la teoría que efectivamente tenga entre el acervo de su aporte conceptual, a las adaptaciones (en el sentido no histórico). Quizás el caso es analogable al de partícula según Moulines. No conozco de ninguna teoría que determine el concepto de adaptación, y creo que puede afirmarse que tal teoría no ha sido tenida en cuenta, "probablemente porque la misma tiene una estructura tan elemental que nadie se preocupa por explicitarla” (Moulines 1991, p. 224). Esto explica lo fácil (casi natural) que resulta pensar que se trata de un dato genuino (desprovisto de carga teórica) y que podemos determinar adaptaciones sin el auxilio de teoría alguna. 
Chomsky 2016). Aunque ambos coincidan en este diagnóstico, Wallace -se dice- terminó cortando el nudo gordiano, y adoptando una forma (laxa) de creacionismo; mientras que Darwin abrió la puerta a otros mecanismos evolutivos. (Más sobre esta interpretación en Gould 1980.)

El punto es que, teniendo en cuenta las reconstrucciones anteriores, uno debe elucidar en qué estaban pensando cuando hablaban de selección natural antes de sacar una conclusión respecto de sus respectivas posiciones. Ya insinuamos que por "selección natural" se puede entender:

(1) el proceso evolutivo en el que la emergencia de la variación, la selección en sí, y la transmisión hereditaria, son tomadas conjuntamente; o

(2) la lucha por la supervivencia (a la luz de lo dicho anteriormente, el lector tendrá ya sus reservas para con esta interpretación); o bien

(3) una (ahora así llamada por nosotros) colección de elementos teóricos en la cual el éxito en la supervivencia no es más que uno de ellos.

No siempre está claro (incluso en el mismo Darwin) sobre cuál de estas cosas se habla cuando se dice que la selección natural ha de ser aplicada a un caso específico.Dado que Wallace nunca aceptó la alternativa de la heredabilidad de los caracteres adquiridos, uno bien puede sospechar que tanto él como Darwin estaban pensando en la tercera acepción (sin la nomenclatura, por supuesto), es decir, en la teoría como un todo. Sin embargo, la situación no está tan clara cuando uno hecha una segunda mirada y ve qué cosa es la que Wallace se muestra remiso a aceptar en "oposición” a la selección natural. Dice Wallace en su Darwinism:

Aunque mantengo, e incluso lo hago con mayor fuerza que antes, mis diferencias con algunas de las observaciones de Darwin, mi trabajo entero tiende por la fuerza a ilustrar la abrumadora importancia de la Selección Natural sobre todos los otros agentes en la producción de nuevas especies. Así, hago mía a la anterior posición de Darwin, desde la cual retrocedió en ediciones posteriores de sus obras, a causa de las críticas y objeciones que procuré mostrar que eran en realidad poco sólidas. Aun en el rechazo a la fase de selección sexual como dependiente de la elección femenina, insisto en la mayor eficacia de la selección natural. Esta es la doctrina Darwiniana preeminente, y por lo tanto reclamo para mi libro la posición de un defensor del Darwinismo puro. (Wallace 1889, p. 5, énfasis nuestro; ver también Wallace 1870)

Notemos entonces que la dicotomía involucra a la selección sexual y a la selección natural. Veamos ahora un párrafo de Darwin en su The Descent of Man que refleja el "retroceso" al que aludía Wallace:

Tal vez atribuí demasiado a la acción de la selección natural o supervivencia del más apto [...]. Al comienzo no consideré lo suficiente la existencia de estructuras que, hasta donde podemos juzgar hoy, no son ni beneficiosas ni perjudiciales [...]. No fui capaz de anular la influencia de mi anterior creencia, entonces casi universal, de que cada especie había sido creada con un propósito; y esto llevó a mi presuposición tácita de que cada detalle de estructura, a excepción de los rudimentos, era de servicio especial, incluso cuando no se lo reconociera. Naturalmente, cualquiera que tenga en mente esta presuposición extendería en demasía la acción de la selección natural. (Darwin 1871, I, p. 152, énfasis nuestro)

Quiero subrayar aquí dos cuestiones de esta autocrítica de Darwin:

(1) En primer lugar, vemos que Darwin asume aquí un sentido restrictivo de "selección natural" que parece consistente con la que sigue Wallace en la cita anterior: siempre que la selección natural se equipare (inadecuadamente) a la lucha por la supervivencia, aparecerá como rival de la selección sexual.

Estos párrafos sugieren que la disputa entre Wallace y Darwin no estaba dada en ver si era TSN como un todo o bien era una alternativa con tintes lamarckianos (o bien era alguna otra) lo que había que aplicar en el caso en disputa, sino más bien entre dos elementos teóricos especializados, esto es, entre la selección sexual y la selección natural "restringida" de la que hablaba Endler (la lucha por la supervivencia). Ambos estaban de acuerdo en que ésta última no era una buena explicación para la emergencia de ciertos rasgos humanos. Pero si las alternativas son sólo esas, la disputa se da, en nuestra perspectiva, entre dos elementos teóricos de una y la misma teoría (TSN).

Todo esto nos lleva a sostener que hemos de pensar dos veces a la hora de responder a la pregunta respecto del dominio de aplicación de TSN en Darwin. Si la selección sexual es parte de TSN, pues 
entonces tal dominio de aplicación será necesariamente más amplio que lo que uno podría pensar cuando no se tiene en cuenta esta elucidación.

Queda todavía por responder si por esto hemos de concluir que Darwin sostenía o sostuvo en alguna oportunidad que la aplicación de TSN es irrestricta.

(2) En segundo término, notemos que Darwin retrocede de la aplicación de la selección natural allí donde modifica la abundancia de elementos que caerían bajo sus explananda. Recordemos que Darwin nunca pretendió que la selección natural fuera la responsable de la adquisición de cualquier rasgo. La discusión se crea en un subconjunto específico de ellos (mayormente) llamados "adaptaciones". El punto es que el "retroceso" no consiste en que ahora reconozca que el mismo "fenómeno" pueda ser explicado por una teoría alternativa a la selección natural, sino que se negocia la teoría a aplicar porque cambia la forma en que considera al "fenómeno" del que se quiere dar cuenta: si ya no hay adaptación, no estamos obligados a adoptar la selección natural, es decir, sin explanandum, la apelación al explanans pierde sentido.

Ahora bien, ¿significa eso que TSN debe ser aplicada irrestrictamente ante toda adaptación? Sí y no. Sí, en el sentido intencional (en tanto que adaptación, es un $M_{p p}$-más precisamente, un miembro del conjunto I- de TSN), pero eso no significa que Darwin diera por hecho que una vez identificada una adaptación, la misma constituiría un caso de aplicación exitosa de TSN (no toda adaptación es un modelo efectivo -M- de TSN).

Dicho de este modo, parece que en lo que Darwin y Wallace no coincidían era más bien en la extensión del conjunto de objetos que caen bajo el concepto de "adaptación”, y no en el hecho de que una vez que hemos dado con una adaptación, pensemos en ella como un "candidato" a modelo efectivo de TSN. Se trata más bien de una disputa respecto de la naturaleza de las "parcelas del mundo", más que de la teoría que hay que aplicar (independientemente de que la aplicación resulte exitosa o no) una vez identificadas tales parcelas como "adaptaciones".

Esto nos lleva a un segundo nivel en el que también hay un claro disenso: hay ciertas adaptaciones, en particular, en el hombre, que no constituyen una aplicación exitosa de TSN para Wallace y sí para Darwin. Pero eso no es una divergencia en cuanto a las aplicaciones pretendidas de TSN sino más bien de su éxito aplicativo. Wallace quitaría a esas adaptaciones del dominio de TSN por considerar que su aserción empírica, para ese sistema particular, es falsa, cosa que Darwin no haría.

Esto no quiere decir que Darwin pensara siempre en TSN a la hora de explicar el origen de todas las adaptaciones. ${ }^{10} \mathrm{El}$ punto es que en lo atinente a la disputa sobre ciertos elementos específicos del dominio de aplicación de su teoría, no hay evidencia textual en la que Darwin oponga TSN a otros mecanismos (como por ejemplo, el uso y el desuso en conjunción con la herencia de caracteres adquiridos) para explicar el origen de aquellos mismos elementos que sí generaban dudas en Wallace (es importante que en esta cuestión quede claro que la heredabilidad de los caracteres adquiridos difiere de TSN de un modo que la lucha por la existencia no difiere de la lucha por emparejarse). Allí (en aquellos rasgos) donde Wallace duda del éxito de TSN, Darwin no lo hace, independientemente de que apele o no al "auxilio" adicional de otros mecanismos.

Notemos esta cita de Darwin a modo de ejemplo:

Dado que el hombre está propenso, como cualquier otro animal, a experimentar múltiples diferencias individuales o sutiles variaciones, y sin dudas así sucedió con anteriores ancestros del hombre; las variaciones siendo tanto entonces como ahora inducidas por las mismas causas generales y gobernadas por las mismas leyes complejas y generales. Y dado que todos los animales tienden a multiplicarse más allá de sus medios de subsistencia, entonces debe haber habido entre los progenitores del hombre una inevitable lucha por la existencia y selección natural. Este proceso habrá sido auxiliado grandemente por los efectos hereditarios del mayor uso de las partes; estando estos dos procesos actuando incesantemente entre sí. También parece [...] que varios caracteres sin importancia han sido adquiridos por el hombre a través de la selección sexual. Un cambio residual sin explicación, tal vez importante, debe asumirse

\footnotetext{
${ }^{10}$ Recordemos, por ejemplo, las referencias en las que Darwin ni siquiera menciona a la selección natural para la emergencia de ciertos rasgos, o bien lo hace, pero haciéndole tomar un protagonismo "posterior", ante la co-opción por parte de tales rasgos de una funcionalidad diferente a la que le dio origen, si es que esta última existió (lo que luego se llamaría exaptación, ver Gould \& Vrba 1982).
} 
como estando a merced de la acción uniforme de agentes desconocidos, los cuales ocasionalmente inducen desviaciones muy marcadas y abruptas en la estructura de nuestras producciones domésticas (Darwin 1871, I, p. 154; comparar con Darwin 1872, pp. 159-164)

Tanto en Darwin como en Wallace hay una exclusión de rasgos del conjunto I, pero por diferentes razones: Darwin los quita en tanto ya no los reconoce como adaptaciones y por lo tanto ya no tiene sentido preguntarse si podrían ser (siempre en terminología estructuralista) "modelos efectivos" de la teoría. Wallace, por su parte, los quita porque cree que si bien su candidatura a "modelos efectivos" sigue en pie, fracasan en ser aplicaciones exitosas de la teoría, y por lo tanto -para esos casos particulares- la teoría es falsa y debe ser descartada. (Más al respecto de la relación entre Darwin y Wallace en lo que refiere a TSN, en Ginnobili \& Blanco 2010.)

Naturalmente, y en una cuestión que aquí sólo dejo planteada, TSN no sólo experimentó cambios del conjunto I por exclusión, sino también por incorporación. La teoría es lo suficientemente versátil y fértil como para ser aplicada en casos de distinto tipo en relación a aquellos para los que fue formulada originalmente. ${ }^{11}$

\section{Relevancia de la "caída" transtemporal de ejemplares}

Otra forma en la que se puede documentar el "desarrollo" de una teoría consiste en mostrar variaciones en estratos especializados.

Tomemos un ejemplo concreto: la cola del pavo real. Dice Darwin:

Dado que las aves [los pavos reales] siempre se reproducen cuando la comida es abundante, los machos probablemente no sufren mucho la inconveniencia que acarrea la búsqueda de comida con la capacidad reducida de movimiento con la que cuentan, pero no puede haber duda alguna de que ellos deben ser más propensos a culminar como víctimas de las aves de presa. Ni puede haber duda alguna de que la larga cola del pavo real [...] debe convertirlo en una presa más fácil para el tigre merodeador. (Darwin 1882, p. 403)

La cola del pavo real es neutral respecto del encuentro de comida, desventajosa a la hora de esconderse y/o huir de los predadores, y, a la vez, una enorme ventaja en otro sitio. Dice Darwin en la misma obra:

Ornamentos de toda clase, independientemente de que fueran obtenidas temporal o permanentemente, son desplegados seductoramente por los machos, y aparentemente sirven para excitar, atraer, o fascinar a las hembras (Darwin 1882, p. 394).

Veamos cómo aparece esto en un texto para profesores de Biología:

La selección sexual es un "caso especial" de selección natural. La selección sexual actúa sobre la habilidad de un organismo para obtener (a menudo por cualquier medio que fuera necesario) la cópula exitosa con un compañero/a. La selección hace que muchos organismos vayan a extremos para el sexo: los pavos reales sostienen colas elaboradas [...]. La selección sexual a menudo es lo suficientemente fuerte como para producir caracteres que son nocivos para la sobrevida del individuo. Por ejemplo, las plumas de las colas extravagantes y coloridas probablemente atraen a predadores como así también a miembros interesados del sexo opuesto. (The Understanding Evolution for Teachers, http://evolution.berkeley.edu/evosite/evo101/IIIE3Sexualselection.shtml; ver Petrie, Halliday \& Sanders 1991)

\footnotetext{
${ }^{11} \mathrm{Al}$ decir de Kuhn:
}

En esta aplicación común, el paradigma funciona, permitiendo la renovación de ejemplos cada uno de los cuales podría servir para reemplazarlo. Por otra parte, en una ciencia, un paradigma es raramente un objeto para renovación. En lugar de ello, tal y como una decisión judicial aceptada en el derecho común, es un objeto para una mayor articulación y especificación, en condiciones nuevas o más rigurosas (Kuhn 1962, p. 51). 
Así, si hemos de explicar el origen de la cola de los pavos reales, no apelamos a la lucha por la vida, sino a la teoría de la selección sexual, en este caso, por la atracción por parte de los machos de compañeras sexuales.

Sin embargo, un estudio publicado en febrero de 2008 en Animal Behaviour (Takahashi et al. 2008) sobre el tema, fruto de un esfuerzo de siete años de investigación, muestra que en la actualidad no se da una ventaja reproductiva por razones de selección sexual por parte de las hembras en virtud de lo elaborado (o, al menos en virtud de lo que nosotros consideramos "elaborado", es decir, en virtud de aquello que atraería depredadores: tamaño, colorido, número de ocelos, y simetría) de las plumas de la cola de los machos. Además, tampoco los predadores parecen mostrar preferencias por pavos de colas mayores.

¿Qué hacer frente a esto?

El camino convencional frente a este escenario es el de no desesperar, pensar en otro criterio de aptitud, de modo que el que no se de una aplicación exitosa de la teoría en relación a una especialización particular (en este caso la atracción de hembras dentro de la selección sexual) nos llevará o bien a verla como una aplicación pretendida de otro elemento teórico especializado; o bien, si se falla en todos los casos disponibles, y dado que el conjunto de especializaciones también es abierto, nos llevará a encontrar una nueva especialización que enriquezca a esta misma teoría para la cual el caso sea una aplicación exitosa; e incluso si tal cosa no es posible, nos llevará en última instancia a rendirnos y a excluir al caso como parte del conjunto I de TSN como un todo (ver Stegmüller 1986, p. 78). ${ }^{12}$

Retomando el tema de las aplicaciones exitosas en teorías históricas, en tales casos la aserción empírica de la teoría no consiste en hablar de una parcela de la "realidad" contemporánea, sino de las razones para la emergencia histórica de ese rasgo particular (después de todo, el que la selección natural actúe continuamente en la naturaleza -sin referencia a la emergencia de rasgos- no era una novedad para autores cuyos escritos precedieron a los de Darwin tales como los de Patrick Matthew (1831, ver Wells 1973, Dempster 1983), E. Blyth (1835, 1837), Lyell (1830-1833) o el mismo Paley (1802, pp. $321,323-324) .{ }^{13}$ Así, el que hoy las colas con determinada ornamentación no atraigan hembras no prueba que la cola del pavo real no evolucionara porque atraía hembras, sino que evidencia que en la actualidad no hay una evolución direccional en lo que a este rasgo refiere (al menos no por esas razones). Una vez más, es muy complicado poder saber las razones selectivas para la evolución de un rasgo, y a esto me refería antes (sección 3.1) cuando hablaba de la dificultad a la hora de identificar aplicaciones exitosas en este tipo de teorías, independientemente de que mayormente los científicos parecen verse satisfechos con este tipo de explicaciones.

\section{Conclusiones}

1. Una perspectiva jerárquica de las teorías demanda la identificación y distinción de las modificaciones accidentales que, aun en su relevancia, no afectan la identidad de la teoría.

2. Dado que todo ejemplar es antes una aplicación exitosa, y que los criterios para reconocer una aplicación exitosa están determinados socialmente por la comunidad científica, acordaremos también con que tales criterios no son los mismos cuando TSN es aplicada a un caso no histórico (por ejemplo, en la aserción: "la cola de los pavos reales machos es objeto de selección sexual por parte de las

\footnotetext{
${ }^{12}$ Notemos que esta heurística, que en otro marco podría resultar sospechoso (recuérdese lo dicho al respecto en la sección 2.1), resulta saludable a la luz de una elucidación jerárquica de la teoría, según la cual la ley fundamental nos da un marco dentro del cual pueden formularse nuevas leyes especiales. En términos de Moulines, constituye en este caso un "principio-guía" (Moulines 1978). Como vemos, la eliminación de una aplicación pretendida particular por el fracaso predictivo de la teoría para con ella no basta para refutar a la teoría como un todo (remítase esto a la discusión entre Popper y Kuhn respecto de la falsabilidad de las teorías científicas).

${ }^{13}$ Así como ni Darwin ni Wallace fueron los primeros evolucionistas de la historia, tampoco fueron los primeros en pensar en la selección natural como su mecanismo, o en acuñar el término. Matthews, por ejemplo, vinculó causalmente al transformismo con la selección natural. Sin embargo, restringió su poder a un "halo" esencial, un "vallado" infranqueable propio de la concepción fijjista típica de la época. Así (y salvo quizá el caso de Blyth), varios autores señalaron a la selección natural como responsable de la restricción del cambio (Eiseley 1979). La selección natural era una fuerza conservadora que eliminaba ineptos podando imperfecciones, pero no creaba adaptaciones.
} 
hembras") que cuando es aplicada a un caso histórico (por ejemplo, en la aserción: "la cola de los pavos reales machos adquirió su configuración actual por mediación de la selección sexual”). La importancia de procurarse una solución a este problema no es menor porque si bien es posible determinar la variación (por salida o entrada) de los componentes de $I$, sin que importe la identificación de los miembros de $I_{0}$, sí es importante conocer la extensión de $I_{0}$ para saber si estamos frente a un cambio esencial o a uno meramente accidental. ${ }^{14}$

3. Desde el mismo comienzo, TSN incorporó y excluyó elementos del dominio de aplicación de su elemento teórico básico, aunque esto sucediera en Wallace y Darwin -sus cofundadores- por razones diferentes. Si consideramos que la interpretación de la discusión entre ellos expuesta aquí a la luz de la elucidación estructuralista de la teoría es correcta, tal vez podamos tomar a dicha interpretación como un pequeño botón de muestra de una humilde respuesta al llamado de Wolfgang Stegmüller a construir puentes entre el enfoque sistemático y el enfoque histórico de la ciencia (ver Pérez Ransanz 1999, p. 247).

Bibliografía

Balzer, W., Moulines, C. U. y J. D. Sneed (1987), An Architectonic for Science. The Structuralist Program, Dordrecht: Reidel. (Versión castellana de Pablo Lorenzano: Una arquitectónica para la ciencia. El programa estructuralista, Bernal: Universidad Nacional de Quilmes, 2012.)

Barret, P., Gautey, P., Herbert, S., Kohn, D. y S. Simth (eds.)(1987), Charles Darwin's Notebooks, 1836-1844, Cambridge: Cambridge University Press.

Berwick, R. y N. Chomsky (2016), Why Only Us. Language and Evolution, Cambridge: MIT Press.

Blanco, D. (2008) "La naturaleza de las adaptaciones en la teología natural británica: análisis historiográfico y consecuencias metateóricas”, Ludus Vitalis 16(30): 3-26.

Blanco, D. (2011) "La teología natural y los 'hechos' de la teoría evolutiva”, en Stefano, W. y M. Medhat Pechliye (eds.), Filosofia e História da Biologia, Sâo Pablo: MackPesquisa, pp. 7-44.

Blyth, E. (1835), "An Attempt to Classify the 'Varieties' of Animals”, The Magazine of Natural History 8: 40-53.

Blyth, E. (1837), "On the Psychological Distinctions Between Man and All Other Animals", The Magazine of Natural History n.s. 1: 1-9; 77-85, 131-141.

Caplan, A. L. (1977), “Tautology, Circularity, and Biological Theory”, The American Naturalist 111(978): 390-393.

Caponi, G. (2011), La segunda agenda darwiniana, México: CEFPSVLT.

Castrodeza, C. (1977), "Tautologies, Belief, and Empirical Knowledge in Biology", The American Naturalist 111(978): 393-394.

Cornell, J. (1987), “God's Magnificent Law: The Bad Influence of Theistic Metaphysics on Darwin's Estimation of Natural Selection", Journal of the History of Biology 20(3): 381-412.

Darwin, C. (1859), On the Origin of Species by means of Natural Selection, or the Preservation of Favoured Races in the Struggle for Life, London: John Murray, $6^{\text {a }}$ ed. 1872.

Darwin, C. (1868), The Variation of Animals and Plants under Domestication, London: John Murray.

Darwin, C. (1871), The Descent of Man, and Selection in Relation to Sex, London: John Murray, 2ª ed. 1882.

Dempster, W. (1983), Patrick Matthew and Natural Selection, Edinburgh: Paul Harris Publishing.

Dennett, D. (2007), Romper el hechizo. La religión como fenómeno natural, Madrid: Katz.

\footnotetext{
${ }^{14}$ Moulines (2002) apunta que difícilmente pueda encontrarse una teoría de nulo valor empírico en cualquier momento de su historia, es decir, para la que nunca existieran aplicaciones exitosas $(I \cap r(M) \neq \varnothing)$, y por supuesto que no es este el caso de TSN. Ya vimos (nota 7) que al menos los ejemplares pertenecen a ese conjunto de aplicaciones exitosas; es decir, $I_{0} \subseteq(I \cap r(M))$. Como sea, la determinación de la extensión del conjunto $I_{0}$ de TESN es una cuestión que excede los propósitos de esta contribución.
} 
Díez, J. A. y P. Lorenzano (2002), "La concepción estructuralista en el contexto de la filosofía de la ciencia del siglo XX”, en Díez, J. A. y P. Lorenzano (eds.), Desarrollos actuales de la metateoría estructuralista: problemas y discusiones, Quilmes: Universidad Nacional de Quilmes/Universidad Autónoma de Zacatecas/Universidad Rovira i Virgili, pp. 13-78.

Eiseley, L. (1979), Darwin and the Mysterious Mr. X, New York: Harvest Books.

Endler, J. (1986) Natural Selection in the Wild. Princeton: Princeton University Press.

Falguera, J. (2012), “Leyes fundamentales a priori relativizados y géneros”, Stoa 3(5): 65-85.

Ginnobili, S. (2006), La teoría de la selección natural darwiniana, Tesis de licenciatura, Buenos Aires: Universidad de Buenos Aires.

Ginnobili, S. (2010), La estructura de la Teoria de la selección natural. Elucidación de sus conceptos fundamentales, reconstrucción de su estructura y consecuencias del análisis sobre algunas discusiones metateóricas en su alrededor, Tesis doctoral, Buenos Aires: Universidad de Buenos Aires.

Ginnobili, S. y C. Carman (2008), "Deferentes, epiciclos y adaptaciones”, Filosofia e História da Ciencia no Cone Sul. Seleção de Trabalhos do 5 Encontro, Campinas: AFHIC, pp. 399-408.

Ginnobili, S. y D. Blanco (2010), "Wallace y la selección natural (en teoría)", en García, P. y A. Massolo (eds.), Epistemología e Historia de la Ciencia. Selección de Trabajos de las XIX Jornadas, vol. 16, Córdoba: Facultad de Filosofía y Humanidades de la Universidad Nacional de Córdoba, pp. 251-259.

Gould, S. (1980), "Wallace’s Fatal Flaw”, Natural History 89(1):26-40.

Gould, S. y E. Vrba. (1982), “Exaptation - a Missing Term in the Science of Form”, Paleobiology 8(1): 4-15.

Krimbas, C. (1984), “On Adaptation, Neo-Darwinian Tautology, and Population Fitness”, Evolutionary Biology 17: 1-57.

Kuhn, T. S. (1962), The Structure of Scientific Revolutions, Chicago: University of Chicago Press.

Loehle, C. (1988), "Philosophical Tools: Potential Contributions to Ecology”, Oikos 51: 97-104.

Lorenzano, P. (2005a), “Comentarios a 'Explicación teórica y compromisos ontológicos: un modelo estructuralista', de C. Ulises Moulines”, Enrahonar 37: 55-59.

Lorenzano, P. (2005b), “Ejemplares, modelos y principios en la genética clásica”, Scientiae Studia 3(2): 185-203.

Lorenzano, P. (2007), “Leyes fundamentales y leyes de la biología”, Scientiae Studia 5(2): 185-214.

Løvtrup, S. (1987), “The Theoretical Basis of Evolutionary Thought”, Annales des Sciences Naturelles 8: 219-236.

Løvtrup, S. (1990), “Is Physics Different from Biology?”, Rivista di Biologia 83: 455-491.

Lyell, C. (1830-1833), Principles of Geology, en Secord, J. (ed.), Charles Lyell's Principles of Geology (1830-33), London: Penguin Books.

Manser, A. (1965), “The Concept of Evolution”, Philosophy 40: 18-34.

Matthew, P. (1831), Naval Timber and Arboriculture, Edinburgh: Adam and Charles Black.

Mivart, G. (1898), The Groundwork of Science, New York: John Murray.

Moulines, C. U. (1978), “Cuantificadores existenciales y principios-guía en las teorías físicas”, Crítica 10(29): 59-88.

Moulines, C. U. (1986), "Redes teóricas”, en Rolleri, J. (ed.) Estructura y desarrollo de las teorías científicas, México: Universidad Nacional Autónoma de México, pp. 299-324.

Moulines, C. U. (1991), Pluralidad y recursión, Madrid: Alianza.

Moulines, C. U. (1996), "Structuralism: The Basic Ideas", en Balzer, W. y C. U. Moulines (eds.), Structuralist Theory of Science, New York: Walter de Gruyter, pp. 1-13.

Moulines, C. U. (2002), “La concepción estructuralista de la ciencia”, Revista de Filosofía 58: 69-77.

Moulines, C. U. (2011) “Cuatro tipos de desarrollo teórico en las ciencias empíricas”, Metatheoria 1(2): 11-27.

Nelson, P. (1996), “The Role of Theology in Current Evolutionary Reasoning”, Biology and Philosophy 11: $493-517$.

Paley, W. ([1802] 1917), Teología Natural, Dallas: Smith y Lamar. 
Pérez Ransanz, A. R. (1999), Kuhn y el cambio científico, México: Fondo de Cultura Económica.

Peters, R. (1976), "Tautology in Evolution and Ecology”, The American Naturalist 110(971): 1-12.

Petrie, M, Halliday, T. y C. Sanders 1991, "Peahens Prefer Peacocks with Elaborate Tails", Animal Behaviour 41(2): 323-331.

Popper, K. (1963), The Poverty of Historicism, London: Routledge \& Kegan Paul.

Salzano, F. (2000), "Permanence or Change? The Meaning of Genetic Variation”, Proceedings of the National Academy of Science 97: 5317-5321.

Scriven, M. (1959), “Explanation and Prediction in Evolutionary Theory”, Science 130(3374): 477-482.

Stegmüller, W. (1976), “Accidental ('Non-Substantial') Theory Change and Theory Dislodgement: To What Extent Logic Can Contribute to a Better Understanding of Certain Phenomena in the Dynamics of Theories”, Erkenntnis 10: $147-178$.

Takahashi, M., Arita, H., Hiraiwa-Hasegawa, M. y T. Hasegawa (2008), "Peahens do not Prefer Peacocks with more Elaborate Trains", Animal Behaviour 75(4): 1209-1219.

Tuomi, J. (1981), “Structure and Dynamics of Darwinian Evolutionary Theory”, Systematic Zoology 20(1): 22-31.

Tuomi, J. y E. Haukioja (1979), "Predictability of the Theory of Natural Selection: An Analysis of the Structure of the Darwinian Theory”, Savoia 3: 1-8.

Wallace, A. (1870), "Man and Natural Selection”, Nature 3: 8-9.

Wells, K. (1973), “The Historical Context of Natural Selection: The Case of Patrick Matthew”, Journal of the History of Biology 6(2): 225-258.

Williams, M. (1973), "Falsifiable Predictions of Evolutionary Theory", Philosophy of Science 40(4): 518-537. 